\title{
Distribution and antimicrobial resistance profiles of bacterial species in stray cats, hospital-admitted cats, and veterinary staff in South Korea
}

\author{
Woo Kyung Jung \\ Seoul National University College of Veterinary Medicine \\ Sook Shin \\ Seoul National University College of Veterinary Medicine \\ Young Kyung Park \\ Seoul National University College of Veterinary Medicine \\ Suk-Kyung Lim \\ Animal and Plant Quarantine Agency \\ Dong-Chan Moon \\ Animal and Plant Quarantine Agency \\ Kun Taek Park \\ Inje University \\ Yong Ho Park ( $\nabla$ yhp@snu.ac.kr)
}

Seoul National University College of Veterinary Medicine https://orcid.org/0000-0002-4168-6884

\section{Research article}

Keywords: Cat, Antimicrobial resistance, Staphylococcus spp., Enterobacteriaceae, Enterococcus spp.

Posted Date: February 19th, 2020

DOI: https://doi.org/10.21203/rs.2.15626/v3

License: (c) (i) This work is licensed under a Creative Commons Attribution 4.0 International License. Read Full License

Version of Record: A version of this preprint was published at BMC Veterinary Research on April 9th, 2020. See the published version at https://doi.org/10.1186/s12917-020-02326-2. 


\section{Abstract}

Background: Antimicrobial resistance is becoming increasingly important in both human and veterinary medicine. According to the One Health concept, an important step is to monitor the resistance patterns of pathogenic bacteria. In this study, the antimicrobial susceptibility patterns and trends of bacteria isolated from stray cats, hospital-admitted cats, and veterinary staff in South Korea between 2017 and 2018 were investigated

Results : The minimum inhibitory concentrations of different antibiotics for Staphylococcus spp., Enterobacteriaceae, and Enterococcus spp. were determined to establish representatives of different antibiotic classes relevant for treatment or surveillance. For Coagulase-positive and Coagulasenegative Staphylococci, resistance to fluoroquinolones was below $13 \%$, but resistance to ampicillin and penicillin was high (20-88\%). A total of $9.5 \%$, $12.1 \%$, and $40.3 \%$ of staphylococcal isolates from stray cats, hospital-admitted cats, and veterinary staff, respectively, were confirmed to be mecA positive. For Enterobacteriaceae, resistance to carbapenems, fluoroquinolones, and 3 rd generation cephalosporins was low (0-11.1\%). The Enterococcus spp. isolates showed no resistance to vancomycin. The antimicrobial resistance rates of the Staphylococcus spp. and Enterobacteriaceae isolates from stray cats were usually lower than those of isolates from hospital-admitted cats and veterinary staff, but the Enterococcus spp. isolates revealed the opposite. Thus, the antimicrobial resistance varied across bacterial species according to the source from which they were isolated.

Conclusions : Resistance to critically important compounds were low. However, the presence of antimicrobial resistance in cat isolates is of both public health and animal health concern.

\section{Background}

Antimicrobials, including agents of importance to human medicine, are commonly used in companion animal-related veterinary practice [1, 2]. Transmission of antimicrobial resistance among bacteria from animals and veterinarians has been reported [3, 4]. Multidrug-resistant (MDR) Staphylococcus intermedius/pseudintermedius isolates are increasingly being reported to cause problems in small-animal practices [5-7]. According to Rusher et al., wounds and the ear canal are the most common sites for infections with methicillin-resistant $S$. intermedius/pseudintermedius [8]. Knowledge about drug resistance trends over time is important to ensure the long-term efficacy of antibacterial products, and data regarding antimicrobial susceptibility could help veterinarians select the most appropriate antibiotic for treatment purposes. Despite the importance of this aspect, standardized, ongoing surveys regarding antimicrobial resistance are barely available.

Companion animals such as dogs and cats not only share a common environment with humans, but are also administered drugs similar to those prescribed to humans. It has been postulated that companion animals may be an integral part of the transfer of drug resistance, owing to their close and direct contact with humans [9]. All kinds of companion animals (owned and stray animals) are involved in the transmission of drug resistance, even if the particular implication of each animal population has not yet been established clearly [10]. However, most of these concerns have been directed towards parasites of stray cats [11, 12]. Further, in Korea, a large number of studies have focused on dogs, rather than cats [13-15]. The rate of pet ownership is increasing globally, as animals enrich the lives of humans. In Korea, it has been estimated that over $29 \%$ (a total of 5.74 million) of households own a companion animal, with the estimated number of dogs and cats being 6.32 and 2.43 million, respectively [16]. Especially, the total number of companion cats is increasing rapidly, showing a growth of about $41 \%$ in 2017 , compared to the statistic mentioned in a study from 2015 [16]. Stray cats that move freely within urban environments have also been shown to exist, although their population in Seoul has decreased from 200,000 in 2015 to 139,000 in 2017 due to the Trap-Neuter-Return program [17]. Therefore, the systematic control and prevention of drug resistance, through the implementation of a national antimicrobial resistance surveillance method, are greatly needed and should be applied in case of both cats and dogs. This study aimed to determine the antimicrobial susceptibility in the most common bacteria isolated from stray cats, hospital-admitted cats, and veterinary staff.

\section{Results}

Bacterial species

A total of 1,829 Staphylococcus spp., Enterobacteriaceae, and Enterococcus spp. isolates were recovered (281 from stray cats, 978 from hospitaladmitted cats, and 570 from veterinary staff). Table 1 shows the bacterial species isolated based on their origin (stray cats, hospital visiting cats, and veterinary staff). The most frequently isolated pathogens were $E$. coli in case of stray cats and hospital-admitted cats ( $\mathrm{n}=31,11 \%$ and $\mathrm{n}=161,16.5 \%$, respectively) and $S$. epidermidis in case of the veterinary staff $(n=89,15.6 \%)$. Coagulase-negative Staphylococci (CNS) were isolated at a significantly higher $(P<0.05)$ rate from stray cats $(64 / 78,82.1 \%)$ than from hospital-admitted cats $(230 / 350,65.7 \%)$ and veterinary staff $(160 / 294,54.4 \%)$. Among the staphylococcal isolates, $S$. felis was isolated at a significantly higher frequency $(P<0.05)$ from stray cats and hospital-admitted cats $(29 / 74$, $39.2 \%$ and $60 / 264,22.7 \%$, respectively) than from veterinary staff $(6 / 176,3.4 \%)$. On the contrary, the isolation frequency of $S$. epidermidis from veterinary staff $(89 / 176,50.6 \%)$ was significantly higher $(P<0.05)$ than that from stray cats $(6 / 74,8.1 \%)$ and hospital-admitted cats $(39 / 264,14.8 \%)$.

Antimicrobial susceptibility of Staphylococcus spp. 
In case of the isolates of CNS from stray cats, the rates of resistance to ampicillin, penicillin, and oxacillin varied between 20 and $34 \%$, and the rates of resistance to amox/clav, gentamicin, and tetracycline were 6-9\%. Resistance to clindamycin, enrofloxacin, marbofloxacin, and chloramphenicol was absent or low (0-3\%) (Table 3 and Fig. S1). For cephalexin, no CLSI breakpoints are available. The number of isolates of Coagulase-positive Staphylococci (CPS) from stray cats was too small; hence, the resistance rates of these isolates is not being presented.

More than half of the isolates of CPS from hospital-admitted cats showed resistance to ampicillin, penicillin, and amox/clav (59-82\%) (Table 2 and Fig. S1). The rates of resistance to oxacillin, enrofloxacin, marbofloxacin, and tetracycline varied between 12 and $29 \%$, and the rates of resistance to gentamicin, clindamycin, and chloramphenicol were lower (6-9\%). For the isolates of CNS from hospital-admitted cats, the rates of resistance to ampicillin, penicillin, and oxacillin were between 35 and $60 \%$, and the rates of resistance to amox/clav, gentamicin, and tetracycline were $11-24 \%$ (Table 3 and Fig. S1). The rates of resistance to clindamycin, enrofloxacin, marbofloxacin, and chloramphenicol were low (4-7\%) (Table 3 and Fig. $\mathrm{S1}$ ). In case of cephalexin, for which no breakpoints were available, the MIC distributions suggest the presence of acquired resistance for some isolates. The rates of resistance to ampicillin, penicillin, and tetracycline for isolates from hospital-admitted cats were significantly higher $(P<0.05)$ than those for the isolates from stray cats.

In case of the CNS isolates from the veterinary staff, the rates of resistance to ampicillin, penicillin, oxacillin, and tetracycline varied between 59 and $80 \%$, and the rates of resistance to amox/clav and gentamicin were $38 \%$. The rates of resistance to clindamycin, enrofloxacin, marbofloxacin, and chloramphenicol was low (3-11\%) (Table 3 and Fig. S1). In case of cephalexin, for which no breakpoints were available, the MIC distributions suggest the presence of acquired resistance for some isolates, like the case for the isolates of CPS from the hospital-admitted cats. The rates of resistance to ampicillin, penicillin, oxacillin, amox/clav, gentamicin, enrofloxacin, marbofloxacin, and tetracycline for isolates from the veterinary staff were significantly higher $(P<0.05)$ than those for the isolates from stray cats or hospital-admitted cats.

Antimicrobial susceptibility of Enterobacteriaceae

For Enterobacteriaceae isolates from stray cats, resistance to ceftiofur, ceftriaxone, gentamicin, imipenem, meropenem, enrofloxacin, marbofloxacin, and tetracycline was absent or low (0-5\%). The rate of resistance to cefoxitin was shown to be $13 \%$. The rates of resistance to ampicillin, amox/clav, and cephalothin varied between 72 and $100 \%$ (Table 4 and Fig. S1).

For Enterobacteriaceae isolates from hospital-admitted cats, resistance to gentamicin, imipenem, meropenem, enrofloxacin, and marbofloxacin was absent or low $(0-6 \%)$. The rates of resistance to cefoxitin, ceftiofur, ceftriaxone, and tetracycline ranged from 10 to $22 \%$. The rates of resistance to ampicillin, amox/clav, and cephalothin varied between 76 and 99\% (Table 4 and Fig. S1). Tetracycline resistance in isolates from hospital-admitted cats was significantly higher $(P<0.05)$ than that in isolates from stray cats.

For Enterobacteriaceae isolates from veterinary staff, resistance to ceftiofur, ceftriaxone, gentamicin, imipenem, meropenem, enrofloxacin, and marbofloxacin was absent or low (0-8\%). The rates of resistance to cefoxitin and tetracycline were between 36 and $44 \%$, and were significantly higher $(P<0.05)$ than those of isolates from stray cats or hospital-admitted cats. The rates of resistance to ampicillin, amox/clav, and cephalothin varied between $88-98 \%$ (Table 4 and Fig. S1).

Antimicrobial susceptibility of Enterococcus spp.

For Enterococcus spp. isolates from stray cats, rates of resistance to erythromycin, tetracycline, and minocycline were between 51 and $68 \%$; however, resistance to ampicillin, vancomycin and high levels of resistance to streptomycin was absent or low (0-9\%) (Table 5 and Fig. S1). The rates of resistance to penicillin, chloramphenicol, and ciprofloxacin, and high levels of resistance to gentamicin were between 16 and $21 \%$.

For Enterococcus spp. from hospital-admitted cats, rates of resistance to erythromycin, tetracycline, and minocycline were between 39 and $62 \%$; however, resistance to vancomycin and high levels of resistance to streptomycin were absent or low (0-9\%) (Table 5 and Fig. S1). The rates of resistance to ampicillin, penicillin, chloramphenicol, and ciprofloxacin, and high levels of resistance to gentamicin were between 11 and $20 \%$.

For Enterococcus spp. from veterinary staff, rates of resistance to erythromycin, tetracycline, and minocycline were between 25 and $50 \%$; however, resistance to ampicillin, penicillin, vancomycin, chloramphenicol, ciprofloxacin and high levels of resistance to both streptomycin and gentamicin were absent or low (0-10\%) (Table 5 and Fig. S1).

mecA gene detection

In total, 110 (21.4\%) Staphylococcus spp. isolates harbored the mecA gene: 7 (9.5\%) from stray cats, 32 (12.1\%) from hospital-admitted cats, and 71 (40.3\%) from veterinary staff. The rates of $m e c A$ among the staphylococcal isolates from the veterinary staff were higher than those among the staphylococcal isolates from the stray cats or hospital-admitted cats $(P<0.05)$. S. epidermidis was the most prevalent bacterium among the isolates 
from both the hospital-admitted cats and veterinary staff (15/46.9\% and 53/74.7\%, respectively) (data not shown). The oxacillin-resistance rates among the isolates of CNS were higher than those among the isolates of CPS from the hospital-admitted cats and veterinary staff $(P<0.05)$. Generally, the susceptibility ranges of the mecA-containing staphylococci isolated from the stray cats, hospital-admitted cats, and veterinary staff were similar. The rates of resistance to ampicillin and penicillin were between 84.4 and $98.6 \%$, and the rates of resistance to amox/clav, gentamicin, and tetracycline were between 28.6 and $88.7 \%$. Resistance to clindamycin, enrofloxacin, marbofloxacin, and chloramphenicol was absent or low (0$15.5 \%$ ) (Table 6).

Extended spectrum $\beta$-lactamases (ESBL) and carbapenemase detection

Among the 278 Enterobacteriaceae isolates, 1 K. pneumoniae isolate from a stray cat and $1 \mathrm{~K}$. pneumoniae, 1 Enterobacter asburiae, 1 Enterobacter cloacae, and $11 \mathrm{E}$. coli isolates from hospital-admitted cats fulfilled the selection criteria for being termed as ESBL-producing bacteria. Among them, five $E$. coli isolates from hospital-admitted cats were confirmed to possess both $b / a_{\mathrm{TEM}}$ and $b / a_{\mathrm{CTX}-\mathrm{M}}$, while one $K$. pneumoniae isolate from a stray cat and one $K$. pneumoniae isolate from a hospital-admitted cat possessed only bla $a_{\mathrm{TEM}}$. None of the isolates possessed $b / a_{\mathrm{SHV}}$. A total of eight isolates that satisfied the ESBL-producing bacteria selection criteria were negative for all resistance genes tested.

None of the Enterobacteriaceae isolates that were not susceptible to imipenem or meropenem showed enhanced growth in the modified Hodge test.

Phenotypic resistance patterns derived from human clinical breakpoints

Overall, $15 \%$ (9/60) of the isolates of CPS, $25.3 \%$ (115/454) of the isolates of CNS, $48.6 \%(135 / 278)$ of the Enterobacteriaceae isolates, and $27.9 \%$ $(63 / 226)$ of the Enterococcus spp. isolates were susceptible to all antimicrobial agents tested. Additionally, 30\% (18/60) of the isolates of CPS, 33\% (150/454) of the isolates of CNS, $25.5 \%(71 / 278)$ of the Enterobacteriaceae isolates, and $29.2 \%(66 / 226)$ of the Enterococcus spp. isolates showed MDR. The MDR rates of the isolates of CNS obtained from the veterinary staff $(92 / 160,57.5 \%)$ were significantly higher $(P<0.05)$ than those of the isolates of CNS obtained from the stray cats $(7 / 64,10.9 \%)$ or hospital-admitted cats $(51 / 230,22.2 \%)$ (Table 7$)$. The MDR rates of the isolates of CNS obtained from the hospital-admitted cats were also significantly higher $(P<0.05)$ than those of the isolates of CNS obtained from the stray cats. The MDR rates of Enterobacteriaceae isolates obtained from veterinary staff $(22 / 50,44 \%)$ were significantly higher $(P<0.05)$ than those of Enterobacteriaceae isolates obtained from the stray cats $(4 / 39,10.3 \%)$ or hospital-admitted cats $(45 / 189,23.8 \%)($ Table 8$)$. On the contrary, the MDR rates of Enterococcus spp. isolates obtained from the veterinary staff $(1 / 20,5.0 \%)$ were significantly lower $(P<0.05)$ than those of Enterococcus spp. isolates obtained from the stray cats $(18 / 57,31.6 \%)$ or hospital-admitted cats $(47 / 149,31.5 \%)$ (Table 9$)$.

The most common resistance profile among the CNS isolates was BLA-OXA, which was detected in 57 isolates of CNS, while the most common MDR profile was AMG-BLA-BLI-OXA-TET, which was observed in 4 isolates of CNS obtained from the hospital-admitted cats and 24 isolates of CNS obtained from the veterinary staff. Among the Enterobacteriaceae isolates, the most common resistance profile was BLA-BLI-GC, which was detected in 1 isolates of Enterobacteriaceae obtained from the stray cats, 11 isolates from hospital-admitted cats, and 9 isolates from the veterinary staff. In the Enterococcus spp. isolates, the most common resistance profile was MAC-TET, which was detected in 29 isolates of Enterococcus spp., while the most common MDR profile was MAC-PNC-TET, which was observed in 5 isolates of Enterococcus spp. obtained from the stray cats, and 8 isolates from the hospital-admitted cats.

\section{Discussion}

In this study, we report the findings of the first nation-wide cross-sectional study of antimicrobial resistance in Staphylococcus spp., Enterobacteriaceae, and Enterococcus spp. isolates from stray cats, hospital-admitted cats, and veterinary staff in South Korea. The higher detection rate of CNS from stray cats than that among the isolates from hospital-admitted cats and veterinary staff may be caused by the poor, unhygienic surroundings of the stray cats. Among the samples from the veterinary staff, S. epidermidis was the major species isolated, followed by $E$. coli and Serratia marcescens. The most commonly identified staphylococcal species among isolates from both the stray cats and hospital-admitted cats was S. felis, this is in accordance with a previous German study, which showed that half of the Staphylococcus spp. isolates obtained were $S$. felis, but inconsistent with the results of studies in USA and South Africa, which have shown that $S$. intermedius/pseudintermedius and S. epidermidis were the representative staphylococci present in cats [23-25]. S. felis has been regarded as a normal commensal organism present on the skin, the conjunctival sac and eyelid margins, and in the saliva of normal healthy cats, as well as an etiological agent that causes skin infections such as pyoderma and otitis $[26,27]$. On the other hand, the most frequently isolated staphylococcal species among samples from the veterinary staff was $S$. epidermidis, which is consistent with the results of other studies [28,29]. This is presumably due to the different host specificities of the staphylococcal species [4].

Comparison of the results obtained in this study with those from international studies are difficult to interpret due to differences between the study design, drugs tested, breakpoint determination, and temporal or geographic variation. According to the ComPath project of Europe, Pasteurella spp. has been reported to be the major dermatological bacterial pathogen in cats, followed by $S$. intermedius/pseudintermedius and $S$. aureus [30]. For $S$. intermedius/pseudintermedius, the rates of resistance to penicillin, gentamicin, and chloramphenicol were $16-23 \%$, while the rates of resistance to 
oxacillin, amox/clav, enrofloxacin, and marbofloxacin were 8-11\%. In comparison, in case of $S$. aureus, high rates of resistance to penicillin (62\%), as well as low rates of resistance to oxacillin, gentamicin, enrofloxacin, marbofloxacin, and chloramphenicol (0-7\%) were found. In our study, the isolates of CPS which are including both of $S$. intermedius/pseudintermedius and $S$. aureus obtained from the stray cats and hospital-admitted cats showed higher rates of resistance to penicillin, oxacillin, and amox/clav than those observed in case of the isolates analyzed in the ComPath study. However, we observed slightly lower levels of resistance to enrofloxacin and marbofloxacin among isolates of stray cats than those observed in the ComPath study.

The isolates of CNS showed higher rates of resistance to oxacillin than the isolates of CPS from the stray cats, hospital-admitted cats and veterinary staff. This has been previously reported in case of studies on both human and veterinary medicine [4, 31]. The isolates of CPS showed higher resistance than CNS to most of the tested antimicrobials except oxacillin; additionally, the isolates of CPS from stray cats showed significantly higher resistance to ampicillin, penicillin, amox/clav, and tetracycline, and those from hospital-admitted cats showed significantly higher resistance to ampicillin, penicillin, and amox/clav $(P<0.05)$.

In this study, 7 staphylococcal isolates from stray cats and 32 from hospital-admitted cats were identified to harbor the mecA gene; this corresponds to $9.5 \%$ of the isolates from the stray cats and $12.1 \%$ of the isolates from the hospital-admitted cats, respectively. These statistics are similar with those of the ComPath study, which showed that $10.3 \%$ of the isolates from cats harbored the mecA gene [ 30 ]. Among the 176 staphylococcal isolates from the veterinary staff, $71(40.3 \%)$ possessed the $m e c A$ gene; this finding is similar to that of our previous study, which showed that the prevalence of the mecA gene in S. intermedius/pseudintermedius isolates from veterinary staff was $35 \%$ [32].

In an Australian study, 341 clinical E. coli isolates were collected from cats [33]. In this study, lower rates of resistance to cefoxitin, ceftiofur, ceftriaxone, gentamicin, imipenem, enrofloxacin, marbofloxacin, and tetracycline (0-10\%) and higher rates of resistance to ampicillin, amox/clav, and cephalothin (15-100\%) were observed. This is similar to the results of our study, except for the fact that in our study, the rates of resistance to ampicillin, cephalothin, cefoxitin and tetracycline were found to be higher.

Among the Enterococcus spp. isolates, the most prevalent species was E. faecalis, followed by E. faecium; this is in accordance with a German study, which showed that $E$. faecalis represented the vast majority of the Enterococcus spp. isolates (26 of 29 isolates) [25]. No Enterococcus spp. isolates were found to be resistant to vancomycin, a critically important drug whose use is strongly discouraged because it can be considered to be 'reserved for the treatment of serious MRSA infections in humans' [34].

Enterobacteriaceae and Enterococcus spp. were isolated from the samples of veterinary staff only at a low frequency; this may be caused by sampling bias, for e.g., bias due to the fact that only the skin and nasal swab samples were included.

Although limited data are available for comparison, E. coli isolates from hospitalized dogs have been found to be more resistant than those from stray dogs [13]. Similarly, the frequency of the resistance to every antimicrobial tested in this study was higher in case of the isolates of CNS from hospital-admitted cats than in case of those from stray cats; the isolates of CNS from hospital-admitted cats showed significantly higher levels of resistance to penicillin and tetracycline than those from stray cats $(P<0.05)$. The number of isolates of CPS was too small to perform a comparison. The resistance rates of Enterobacteriaceae and Enterococcus spp. isolates from stray cats and hospital-admitted cats were similar. The stray cats tested in this study may be assumed to be healthy cats, because the stray cats was available for the neuter surgery; and, it is true that the hospitaladmitted cats had a greater chance of receiving medication. This could be the reason why the isolates of CNS from hospital-admitted cats showed higher levels of drug resistance. However, why there were no differences between the drug resistance rates of the Enterobacteriaceae and Enterococcus spp. isolates from stray cats and hospital-admitted cats was not understood; this finding has been considered to be a good indicator of the selection pressure caused by the use of antimicrobials [35].

Interestingly, the MDR rates of CNS and Enterobacteriaceae isolates from the veterinary staff were significantly higher than those of the isolates from both the stray cats and hospital-admitted cats $(P<0.05)$. These results support the hypothesis that veterinary staff may serve as possible reservoirs for the dissemination of multidrug resistance in veterinary hospitals. This highlights the importance of vigilance by veterinary staff. Veterinary staff may serve as carriers for the pathogens; in this study, it was observed that the veterinary staff from whom the bacterial isolates were obtained showed no clinical signs of diseases. The MDR rates of CNS and Enterobacteriaceae isolates from the stray cats were significantly lower than that of the isolates from the hospital-admitted cats $(P<0.05)$. This may reflect the fact that stray cats only had a rare opportunity to receive medication. However, it has been known that stray dogs show a higher rate of parasitic infection than housed dogs; this may be due to the scavenging habits of stray dogs, which make them more vulnerable to natural infection than housed dogs [10, 12].

The MDR rate of Enterobacteriaceae isolates from hospital-admitted cats was $23.8 \%$, which was much higher than that seen in case of the Australian study (11.7\% among E. coli isolates from cats) [33].

The ESBL detection rates among the isolates were as follows: $2.6 \%(1 / 39)$ of the isolates from stray cats, 3.2\% (6/189) of the isolates from hospitaladmitted cats, and $0 \%$ of the isolates from veterinary staffs. Among them, five $E$. coli isolates from hospital-admitted cats were confirmed to harbor both bla $a_{\mathrm{TEM}}$ and $b / a_{\mathrm{CTX}-\mathrm{M}}$, while one $K$. pneumoniae isolate from a stray cat and one $K$. pneumoniae isolate from a hospital-admitted cat were observed to harbor only bla $a_{\mathrm{TEM}}$. None of the isolates possessed $b / a_{\mathrm{SHV}}$. Since $2000, \mathrm{CTX}-\mathrm{M} \beta$-lactamases have been identified as the most widespread type of ESBLs, replacing classical TEM- and SHV-type ESBLs [36]. The ComPath study has reported a low prevalence of ESBL-producing bacteria in dogs

Page 5/16 
(2.8\%) and the absence of ESBL-producing bacteria in cats [30]. However, a previous Korean study has shown a higher prevalence of ESBL-producing isolates in dogs and cats (29.2\% in dogs and 13.5\% in cats) [37]. The differences in their prevalence may be caused by the use of an ESBL-selective agar (CHROMagar ESBL). AmpC $\beta$-lactamases were not investigated in this study; they need to be focused on in future studies.

\section{Conclusion}

Staphylococcus spp., and Enterobacteriaceae isolates from stray cats and hospital-admitted cats in Korea showed higher resistance to most of the tested antimicrobials, compared to the isolates from cats in European countries and Australia. Additionally, some isolates showed resistance against antimicrobials that are regarded as critically important for use in humans, such as third-generation cephalosporins and quinolones. It is unclear if the higher resistance to certain kinds of antimicrobials was attributed to a result of misuse of antimicrobials in animal hospitals because the treatment history of cats was not collected in the current study. Nevertheless, considering the risk of cross-transmission of resistant bacteria between cats and humans, emergence and dissemination of antimicrobial resistance in cat will undoubtedly continue to be a challenge in veterinary medicine, from both patient health and public health standpoint. More organized surveillance is required to better understand the mechanism of antimicrobial resistance transmission between veterinary and human medicine, in accordance with the One Health perspective.

\section{Methods}

Sample collection

A total of 2,278 samples were obtained in convenience from 78 stray cats $(n=333), 350$ hospital-admitted cats $(n=1,357)$, and 294 veterinary hospital staff including veterinarians, veterinary technicians, and receptionists ( $n=588$ ) across 20 veterinary hospitals in Seoul, Gangwon-do, Gyeonggi-do, Chungcheong-do, Gyeongsang-do, Jeolla-do, and Jeju-do between 2017 and 2018 (Fig. 1). The stray cats were from the trap-neuter-return program. The captured cats were sent to the local veterinary hospital and the sampling was performed immediately after their arrival. Samples from the 333 stray cats and 1,357 hospital-admitted cats were obtained from the anus $(n=425)$, horizontal ear canal $(n=416), n a s a l$ mucosa $(n=417)$, skin $(n=384)$, and urine $(n=48)$. All samples were collected by skilled veterinarians without anesthesia. The captured stray cats were released after the neutering. The hospital-admitted cats were proceeded to the appropriated treatment after sampling. Human samples were taken from the hand (palm and the skin between fingers; $n=294)$ and nasal cavity $(n=294)$. All samples from the cats and humans were obtained using BD BBL Culture Swabs (BectonDickinson, Sparks, MD, USA), placed on ice, and transported to our lab within $6 \mathrm{~h}$ of their collection.

\section{Bacterial isolation and identification}

The swabs were directly plated on $5 \%$ defibrinated sheep blood agar (Hangang, Gunpo, South Korea) and incubated overnight at $37^{\circ} \mathrm{C}$ for $24 \mathrm{~h}$ under aerobic and anaerobic conditions. All the colonies that showed different morphologies and hemolysis patterns were collected and subjected to MatrixAssisted Laser Desorption Ionization-Time of Flight mass spectrometry (microflex LT/SH spectrometer Bruker, Bruker Daltonics, Bremen, Germany) for their identification. When multiple isolates of a same species were recovered from a given individual, only one isolate was selected for further analysis. Staphylococcus spp., Enterobacteriaceae, and Enterococcus spp. were most frequently isolated, so that these bacteria were mainly investigated in this study. Both Staphylococcus intermedius and S. pseudintermedius were designated as "S. intermedius/pseudintermedius" because of the difficulty associated with their differentiation.

Antimicrobial susceptibility testing

Minimum inhibitory concentrations were determined using the standardized agar dilution methodology, as recommended by the Clinical and Laboratory Standards Institute (CLSI) guidelines [18, 19]. Escherichia coli ATCC 25922, Staphylococcus aureus ATCC 29213, and Enterococcus faecalis ATCC 29212 were included as quality control strains in the tests, as recommended by the CLSI guidelines. The antimicrobial susceptibility data of the isolates were obtained for the following antibiotics: gentamicin from the aminoglycoside except streptomycin (AMG); streptomycin (STM); ampicillin, and penicillin from the $\beta$-lactam (BLA) groups; oxacillin (OXA); amoxicillin/clavulanate (amox/clav, ratio 2:1) from the $\beta$-lactam/ $\beta$ lactamase inhibitor combination (BLI); enrofloxacin, marbofloxacin, and ciprofloxacin from the fluoroquinolones (FQN); clindamycin from the lincosamide (LIN); chloramphenicol from the phenicol (PNC); cephalexin, cephalothin, and cefoxitin from the $1^{\text {st }}$ and $2^{\text {nd }}$ generation cephalosporins (GC); ceftiofur, and ceftriaxone from the $3^{\text {rd }}$ generation cephalosporins (3GC); imipenem, and meropenem from the carbapenems (CPM); minocycline and tetracycline from the tetracyclines (TET); and vancomycin from the glycopeptide (GLP). The concentration ranges indicated in Tables $3-6$. The isolates were defined as MDR if they showed resistance to at least one drug from three or more antimicrobial classes using human clinical breakpoints, as previously described [20].

Data analyses 
The $\mathrm{MIC}_{50}$ and $\mathrm{MIC}_{90}$ values of the tested antimicrobials were determined for Staphylococcus spp., Enterobacteriaceae, and Enterococcus spp., which were mainly isolated. Based on the MICs, the isolates were categorized as susceptible, intermediate, or resistant to the antibiotics for which CLSI veterinary breakpoints [19] or breakpoints based on human data [18] are available. The CLSI susceptibility and resistance breakpoints utilized are indicated in Tables 3-6.

The isolation and antimicrobial resistance rates of each bacterial species from different origins (stray cats, veterinary hospital visiting cats, and veterinary hospital staff) were analyzed and compared by Fisher's exact test or Chi square test. All statistical analyses were performed using MedCalc statistical software ver. 11. 2. 1 (MEDCALC, Acacialaan, Belgium). In all tests, $P$ values less than 0.05 were considered significant.

Detection of the mecA gene

All S. intermedius/pseudintermedius and coagulase-negative Staphylococci (CNS) isolates for which the MICs of oxacillin were $\geq 0.5 \mu \mathrm{g} / \mathrm{ml}$ and all $S$. aureus isolates for which the MICs of oxacillin were $\geq 4 \mu \mathrm{g} / \mathrm{ml}$ [19] were screened by PCR for the presence of the mecA gene according to a method adapted from Oliverira D. C. et al. [21]. Concurrently, the mecA-positive strain S. aureus ATCC 43300 was used as a quality control organism.

Detection of extended spectrum $\beta$-lactamases (ESBLs) and carbapenemase

The mechanisms underlying the resistance of Enterobacteriaceae towards $\beta$-lactams were further characterized. The presence of ESBLs was tested by a combined double-disc test using clavulanic acid and cephalosporin indicators (cefotaxime and cefrazidime), according to the CLSI guidelines [18]. $E$. coli ATCC 25922 and Klebsiella pneumoniae ATCC 700603 were used as a quality control strains for a combined double-disc test. Genotypic characterization of the ESBL-positive strains was achieved by performing PCR to detect the major groups of genes encoding $\beta$-lactamases, including bla $a_{\mathrm{CTX}-\mathrm{M}}, b / a_{\mathrm{SHV}}$, and bla $a_{\mathrm{TEM}}[22]$.

All Enterobacteriaceae strains that were not susceptible to imipenem or meropenem were tested by performing the modified Hodge test according to the CLSI guidelines [18]. This test represents a simple method for detecting carbapenemase-producing Enterobacteriaceae.

\section{Declarations}

\section{Abbreviations}

MIC: Minimum inhibitory concentration; MDR: Multidrug-resistant; CLSI: Clinical and Laboratory Standards Institute; CNS: Coagulase-negative Staphylococci; CPS: Coagulase-positive Staphylococci; AMG: aminoglycosides except streptomycin; STM: streptomycin; BLA: $\beta$-lactam groups; OXA: oxacillin; BLI: $\beta$-lactam/ $\beta$-lactamase inhibitor combinations; FQN: fluoroquinolones; LIN: lincosamides; PNC: phenicols; GC: $1^{\text {st }}$ and $2^{\text {nd }}$ generation cephalosporins; 3GC: $3^{\text {rd }}$ generation cephalosporins; CPM: carbapenems; TET: tetracyclines; GLP: glycopeptides; amox/clav: amoxicillin/clavulanate; ESBL: extended spectrum $\beta$-lactamases

\section{Ethics approval and consent to participate}

The experiment was approved by the Seoul National University Institutional Animal Care and Use Committee (SNU-170809-4) and Seoul National University Institutional Review Board (1608/001-004). Individual written informed consent for the use of samples was obtained from all cat owners and veterinary staff.

\section{Consent for publication}

Not applicable.

\section{Availability of data and materials}

Data are available from the corresponding author on reasonable request.

\section{Competing interests}

The authors declare that they have no competing interests.

\section{Funding}

This work was supported by a grant from the Animal and Plant Quarantine Agency, Ministry of Agriculture and Forestry (Z-1543081-2017-18-03) and the Cooperative Research Program of Center for Companion Animal Research (Project No. PJ013985022019) of Rural Development Administration, South Korea. 
WKJ analyzed MIC data and drafted the manuscript; SS and YKP was responsible for laboratory testing of bacterial isolates; SKL and DCM contributed to the study design; KTP analyzed statistic and drafted the manuscript; YHP contributed to the study design and drafted the manuscript.

\section{Acknowledgements}

The authors wish to thank Seong Mi Nho and Se Ra Shin for their outstanding technical assistance.

\section{References}

1. Buckland EL, O'Neill D, Summers J, Mateus A, Church D, Redmond L, Brodbelt D. Characterisation of antimicrobial usage in cats and dogs attending UK primary care companion animal veterinary practices. Vet Rec. 2019;179(19):489.

2. Pedersen K, Pedersen K, Jensen H, Finster K, Jensen VF, Heuer OE. Occurrence of antimicrobial resistance in bacteria from diagnostic samples from dogs. J Antimicrob Chemother. 2007;60(4):775-781.

3. Youn JH, Hwang SY, Kim SH, Koo HC, Shin S, Lim SK, Park YH. mecA gene transferrability and antibiogram of zoonotic Staphylococcus intermedius from animals, staff and the environment in animal hospitals in Korea. J Microbiol Biotechnol. 2010;20(2):425-432.

4. Moon BY, Youn JH, Shin S, Hwang SY, Park YH. Genetic and phenotypic characterization of methicillin-resistant staphylococci isolated from veterinary hospitals in South Korea. J Vet Diagn Invest. 2012;24(3):489-498.

5. van Duijkeren E, Catry B, Greko C, Moreno MA, Pomba MC, Pyorala S, Ruzauskas M, Sanders P, Threlfall EJ, Torren-Edo J et al. Review on methicillin-resistant Staphylococcus pseudintermedius. J Antimicrob Chemother. 2011;66(12):2705-2714.

6. Lehner G, Linek M, Bond R, Lloyd DH, Prenger-Berninghoff E, Thom N, Straube I, Verheyen K, Loeffler A. Case-control risk factor study of methicillinresistant Staphylococcus pseudintermedius (MRSP) infection in dogs and cats in Germany. Vet Microbiol. 2014;168(1):154-160.

7. Weese JS, van Duijkeren E. Methicillin-resistant Staphylococcus aureus and Staphylococcus pseudintermedius in veterinary medicine. Vet Microbiol. 2010;140(3-4):418-429.

8. Ruscher C, Lubke-Becker A, Wleklinski CG, Soba A, Wieler LH, Walther B. Prevalence of Methicillin-resistant Staphylococcus pseudintermedius isolated from clinical samples of companion animals and equidaes. Vet Microbiol. 2009;136(1-2):197-201.

9. So JH, Kim J, Bae IK, Jeong SH, Kim SH, Lim SK, Park YH, Lee K. Dissemination of multidrug-resistant Escherichia coli in Korean veterinary hospitals. Diagn Microbiol Infect Dis. 2012;73(2):195-199.

10. Sowemimo OA. The prevalence and intensity of gastrointestinal parasites of dogs in lle-lfe, Nigeria. J Helminthol. 2009;83(1):27-31.

11. Dashti A, Santin M, Cano L, de Lucio A, Bailo B, de Mingo MH, Koster PC, Fernandez-Basterra JA, Aramburu-Aguirre J, Lopez-Molina N et al. Occurrence and genetic diversity of Enterocytozoon bieneusi (Microsporidia) in owned and sheltered dogs and cats in Northern Spain. Parasitol Res. 2019;118(10):2979-2987.

12. Fu Y, Huang Y, Abuzeid AMI, Hang J, Yan X, Wang M, Liu Y, Sun Y, Ran R, Zhang P et al. Prevalence and potential zoonotic risk of hookworms from stray dogs and cats in Guangdong, China. Vet Parasitol Reg Stud Reports. 2019;17:100316.

13. Nam HM, Lee HS, Byun JW, Yoon SS, Jung SC, Joo YS, Lim SK. Prevalence of antimicrobial resistance in fecal Escherichia coli isolates from stray pet dogs and hospitalized pet dogs in Korea. Microb Drug Resist. 2010;16(1):75-79.

14. Kang JH, Chung TH, Hwang CY. Clonal distribution of methicillin-resistant Staphylococcus pseudintermedius isolates from skin infection of dogs in Korea. Vet Microbiol. 2017;210:32-37.

15. Chung YS, Hu YS, Shin S, Lim SK, Yang SJ, Park YH, Park KT. Mechanisms of quinolone resistance in Escherichia coli isolated from companion animals, pet-owners, and non-pet-owners. J Vet Sci. 2017;18(4):449-456.

16. Ji I, Kim H, Kim W, Seo G. Development Strategies for the Companion Animal Industry. In: Korea Rural Economic Institute; 2017. P. 11-13.

17. Cho YJ, Lee YA, Hwang BR, Han JS, Hwang JS. Stray cat inhabitation status monitoring in Seoul. In: Seoul city; 2017. P. 59-60.

18. CLSI. Performance Standards for Antimicrobial Susceptibility Testing; Approved Standard. $27^{\text {th }}$ ed. Wayne, Pennsylvania, CLSI; 2017.

19. CLSI. Performance Standards for Antimicrobial Disk and Dilution Susceptivility Tests for Bacteria Isolated from Animals. $3^{\text {rd }}$ ed. Wayne, Pennsylvania, CLSI; 2015.

20. Magiorakos AP, Srinivasan A, Carey RB, Carmeli Y, Falagas ME, Giske CG, Harbarth S, Hindler JF, Kahlmeter G, Olsson-Liljequist B et al. Multidrugresistant, extensively drug-resistant and pandrug-resistant bacteria: an international expert proposal for interim standard definitions for acquired resistance. Clin Microbiol Infect. 2012;18(3):268-281.

21. Oliveira DC, de Lencastre H. Multiplex PCR strategy for rapid identification of structural types and variants of the mec element in methicillinresistant Staphylococcus aureus. Antimicrob Agents Chemother. 2002;46(7):2155-2161.

22. Monstein HJ, Ostholm-Balkhed A, Nilsson MV, Nilsson M, Dornbusch K, Nilsson LE. Multiplex PCR amplification assay for the detection of blaSHV, blaTEM and blaCTX-M genes in Enterobacteriaceae. APMIS. 2007;115(12):1400-1408. 
23. Lane MJ, Roy AF, Kearney MT, Pucheu-Haston CM. Characterization, distribution, antimicrobial resistance and resistance risk factors in staphylococci isolated from cats from 2001 to 2014. Vet Med Sci. 2018;4(4):315-325.

24. Qekwana DN, Sebola D, Oguttu JW, Odoi A. Antimicrobial resistance patterns of Staphylococcus species isolated from cats presented at a veterinary academic hospital in South Africa. BMC Vet Res. 2017;13(1):286.

25. Teichmann-Knorrn S, Reese S, Wolf G, Hartmann K, Dorsch R. Prevalence of feline urinary tract pathogens and antimicrobial resistance over five years. Vet Rec. 2018;183(1):21.

26. Espinola MB, Lilenbaum W. Prevalence of bacteria in the conjunctival sac and on the eyelid margin of clinically normal cats. J Small Anim Pract. 1996;37(8):364-366.

27. Litster A, Moss SM, Honnery M, Rees B, Trott DJ. Prevalence of bacterial species in cats with clinical signs of lower urinary tract disease: recognition of Staphylococcus felis as a possible feline urinary tract pathogen. Vet Microbiol. 2007;121(1-2):182-188.

28. Becker K, Heilmann C, Peters G. Coagulase-negative staphylococci. Clin Microbiol Rev. 2014;27(4):870-926.

29. Xu Z, Shah HN, Misra R, Chen J, Zhang W, Liu Y, Cutler RR, Mkrtchyan HV. The prevalence, antibiotic resistance and mecA characterization of coagulase negative staphylococci recovered from non-healthcare settings in London, UK. Antimicrob Resist Infect Control. $2018 ; 7: 73$.

30. Ludwig C, de Jong A, Moyaert H, El Garch F, Janes R, Klein U, Morrissey I, Thiry J, Youala M. Antimicrobial susceptibility monitoring of dermatological bacterial pathogens isolated from diseased dogs and cats across Europe (ComPath results). J Appl Microbiol. 2016;121(5):12541267.

31. Garza-Gonzalez E, Morfin-Otero R, Llaca-Diaz JM, Rodriguez-Noriega E. Staphylococcal cassette chromosome mec (SCC mec) in methicillinresistant coagulase-negative staphylococci. A review and the experience in a tertiary-care setting. Epidemiol Infect. 2010;138(5):645-654.

32. Youn JH, Yoon JW, Koo HC, Lim SK, Park YH. Prevalence and antimicrogram of Staphylococcus intermedius group isolates from veterinary staff, companion animals, and the environment in veterinary hospitals in Korea. Vet Diagn Invest. 2011;23(2):268-274.

33. Saputra S, Jordan D, Mitchell T, Wong HS, Abraham RJ, Kidsley A, Turnidge J, Trott DJ, Abraham S. Antimicrobial resistance in clinical Escherichia coli isolated from companion animals in Australia. Vet Microbiol. 2017;211:43-50.

34. Hillier A, Lloyd DH, Weese JS, Blondeau JM, Boothe D, Breitschwerdt E, Guardabassi L, Papich MG, Rankin S, Turnidge JD et al. Guidelines for the diagnosis and antimicrobial therapy of canine superficial bacterial folliculitis (Antimicrobial Guidelines Working Group of the International Society for Companion Animal Infectious Diseases). Vet Dermatol. 2014;25(3):163-e143.

35. Caprioli A, Busani L, Martel JL, Helmuth R. Monitoring of antibiotic resistance in bacteria of animal origin: epidemiological and microbiological methodologies. Int J Antimicrob Agents. 2000;14(4):295-301.

36. Ewers C, Bethe A, Semmler T, Guenther S, Wieler LH. Extended-spectrum beta-lactamase-producing and AmpC-producing Escherichia coli from livestock and companion animals, and their putative impact on public health: a global perspective. Clin Microbiol Infect. 2012;18(7):646-655.

37. Hong JS, Song W, Park HM, Oh JY, Chae JC, Shin S, Jeong SH. Clonal Spread of Extended-Spectrum Cephalosporin-Resistant Enterobacteriaceae Between Companion Animals and Humans in South Korea. Front Microbiol. 2019;10:1371.

\section{Tables}

Table 1. Bacterial isolates from 78 stray cats, 350 hospital-admitted cats, and 294 veterinary staff 


\begin{tabular}{|c|c|c|c|c|}
\hline Species identified & & Stray cat (78) & Hospital-admitted cat (350) & Veterinary staff (294) \\
\hline Staphylococcus spp. & & 74 & 264 & 176 \\
\hline \multirow[t]{3}{*}{ Coagulase-positive Staphylococci (CPS) } & S. aureus & 5 & 29 & 10 \\
\hline & S. intermedius/ pseud intermedius & 3 & 5 & 5 \\
\hline & S. hyicus & 2 & & 1 \\
\hline \multirow[t]{20}{*}{ Coagulase-negative Staphylococci (CNS) } & S. capitis & & 7 & 4 \\
\hline & S. caprae & 1 & 2 & \\
\hline & S. chromogenes & 1 & 1 & 1 \\
\hline & S. cohnii & 2 & 5 & 3 \\
\hline & S. condimenti & & 2 & \\
\hline & S. epidermidis & 6 & 39 & 89 \\
\hline & S. equorum & 1 & 8 & 1 \\
\hline & S. felis & 29 & 60 & 6 \\
\hline & S. gallinarum & & & 1 \\
\hline & S. haemolyticus & & 5 & 14 \\
\hline & S. hominis & 3 & 17 & 4 \\
\hline & S. Iugdunensis & & 1 & 5 \\
\hline & S. nepalensis & 1 & 1 & 1 \\
\hline & S. pettenkoferi & & 6 & 1 \\
\hline & S. saprophyticus & & 14 & 9 \\
\hline & S. schleiferi & 3 & 3 & 4 \\
\hline & S. sciuri & 6 & 6 & 1 \\
\hline & S. simulans & 9 & 26 & 6 \\
\hline & S. warneri & 1 & 6 & 7 \\
\hline & S. xylosus & 1 & 21 & 3 \\
\hline Enterobacteriaceae & & 39 & 189 & 50 \\
\hline Citrobacter braakii & & 1 & 1 & \\
\hline Citrobacter spp. & & 1 & 4 & \\
\hline Enterobacter aerogenes & & & 4 & 12 \\
\hline Enterobacter asburiae & & & 2 & \\
\hline Enterobacter cloacae & & 1 & 2 & \\
\hline Enterobacter hormaechei & & 1 & & \\
\hline Enterobacter kobei & & & & 1 \\
\hline Enterobacter ludwigii & & & 2 & \\
\hline Enterobacterspp. & & 1 & & \\
\hline Escherichia coli & & 31 & 161 & 18 \\
\hline Klebsiella pneumoniae & & 2 & 4 & 2 \\
\hline Klebsiella variicola & & & 4 & \\
\hline Serratia liquefaciens & & & 1 & \\
\hline Serratia marcescens & & & 1 & 17 \\
\hline Serratia spp. & & 1 & & \\
\hline Enterococcus spp. & & 57 & 149 & 20 \\
\hline Enterococcus avium & & 3 & 5 & \\
\hline Enterococcus casseliflavus & & 2 & 2 & 2 \\
\hline Enterococcus durans & & & & 1 \\
\hline Enterococcus faecalis & & 25 & 74 & 9 \\
\hline Enterococcus faecium & & 13 & 38 & 5 \\
\hline Enterococcus gallinarum & & 4 & 7 & \\
\hline Enterococcus hirae & & 10 & 22 & 3 \\
\hline Enterococcus saccharolyticus & & & 1 & \\
\hline Others & & 74 & 215 & 45 \\
\hline Total & & 281 & 978 & 570 \\
\hline
\end{tabular}

Table 2. MICs of 11 antimicrobial agents against 57 Coagulase-positive Staphylococci ${ }^{1}$ isolated in this study 


\begin{tabular}{|c|c|c|c|c|c|c|c|c|c|c|c|c|c|c|c|c|}
\hline \multirow[t]{2}{*}{ Antimicrobials } & \multirow{2}{*}{$\begin{array}{l}\mathrm{MIC}_{50} \\
(\mu \mathrm{g} / \mathrm{ml})\end{array}$} & \multirow{2}{*}{$\begin{array}{l}\mathrm{MIC}_{90} \\
(\mu \mathrm{g} / \mathrm{ml})\end{array}$} & \multicolumn{12}{|c|}{ MIC values $(\mu \mathrm{g} / \mathrm{ml})$} & \multirow[b]{2}{*}{$\% 1$} & \multirow[b]{2}{*}{$\% \mathrm{~S}$} \\
\hline & & & 0.008 & 0.0150 .03 & 0.06 & 0.12 & 0.25 & 0.5 & 1 & 2 & 8 & 16 & $\begin{array}{ll}64 & 128\end{array}$ & $\% \mathrm{R}$ & & \\
\hline Ampicillin ${ }^{2}$ & $1 / 2 / 1^{3}$ & $8 / 16 / 4$ & & $0 / 2 / 0$ & $1 / 1 / 0$ & $2 / 4 / 2$ & $0 / 2 / 2$ & $1 / 2 / 2$ & $1 / 4 / 2$ & $2 / 9 / 6 \overline{1 / 3 / 1}$ & $1 / 1 / 0$ & \multicolumn{2}{|c|}{$\overline{0 / 4 / 1} \overline{1 / 1 / 0} \overline{0 / 1 / 0}$} & $70 / 74 / 69$ & $0 / 0 / 0$ & $30 / 27 / 31$ \\
\hline Penicillin ${ }^{4}$ & $1 / 2 / 2$ & $16 / 16 / 16$ & $1 / 0 / 0$ & $0 / 1 / 0$ & $1 / 2 / 0$ & $1 / 3 / 2$ & $0 / 2 / 1$ & $0 / 3 / 1$ & $2 / 3 / 3$ & $1 / 6 / 40 / 6 / 2$ & $2 / 1 / 0$ & \multicolumn{2}{|c|}{$1 / 4 / 20 / 2 / 11 / 1 / 0$} & $70 / 82 / 88$ & $0 / 0 / 0$ & $30 / 18 / 13$ \\
\hline Oxacillin ${ }^{4}$ & $0.25 / 0.5 / 0.5$ & $32 / 16 / 4$ & & & $2 / 1 / 0$ & $1 / 2 / 2$ & $2 / 4 / 2$ & $2 / 17 / 6$ & $1 / 3 / 1$ & $0 / 3 / 20 / 0 / 2$ & & \multicolumn{2}{|c|}{$0 / 1 / 02 / 1 / 10 / 2 / 0$} & $20 / 21 / 29$ & $0 / 0 / 0$ & $80 / 80 / 65$ \\
\hline Amox/clav & $1 / 1 / 0.5$ & $2 / 4 / 4$ & & $2 / 2 / 0$ & & $0 / 3 / 1$ & $1 / 6 / 6$ & $1 / 3 / 1$ & $4 / 14 / 6$ & $51 / 2 / 00 / 0 / 2$ & $1 / 0 / 0$ & \multicolumn{2}{|c|}{$0 / 1 / 0$} & $60 / 59 / 50$ & $10 / 9 / 6$ & $30 / 32 / 44$ \\
\hline Cephalexin $^{5}$ & $4 / 4 / 4$ & $64 / 16 / 8$ & & & & & & $1 / 0 / 0$ & $1 / 2 / 0$ & $3 / 2 / 41 / 17 / 9$ & $2 / 8 / 1$ & $0 / 2 / 0$ & \multicolumn{2}{|l|}{$2 / 2 / 10 / 1 / 0$} & & \\
\hline Gentamicin ${ }^{4}$ & $0.25 / 0.5 / 0.5$ & $0.5 / 8 / 32$ & & & $3 / 4 / 2$ & & $3 / 2 / 1$ & $3 / 17 / 8$ & $0 / 4 / 2$ & $0 / 3 / 0$ & $0 / 2 / 1$ & \multicolumn{2}{|c|}{$0 / 1 / 01 / 1 / 2$} & $10 / 6 / 13$ & $0 / 6 / 6$ & $90 / 88 / 81$ \\
\hline Clindamycin ${ }^{2}$ & $0.12 / 0.12 / 0.121$ & $1 / 0.5 / \geq 64$ & & & $2 / 10 / 2$ & $24 / 20 / 9$ & $1 / 0 / 0$ & $0 / 1 / 0$ & $2 / 0 / 0$ & $0 / 0 / 2$ & & $1 / 0 / 1$ & $0 / 3 / 2$ & $10 / 9 / 19$ & $20 / 0 / 13$ & $70 / 91 / 69$ \\
\hline Enrofloxacin & $0.12 / 0.25 / 0.25$ & $0.25 / 4 / 4$ & & $1 / 0 / 1$ & $2 / 6 / 0$ & $5 / 10 / 5$ & $1 / 11 / 6$ & $0 / 3 / 1$ & $0 / 0 / 1$ & $1 / 0 / 00 / 2 / 1$ & & \multicolumn{2}{|c|}{$0 / 1 / 00 / 0 / 10 / 1 / 0$} & $0 / 12 / 13$ & $10 / 0 / 6$ & $90 / 88 / 81$ \\
\hline Marbofloxacin & $0.25 / 0.5 / 0.25$ & $0.5 / 4 / 4$ & & $1 / 0 / 1$ & & $1 / 0 / 0$ & $6 / 15 / 8$ & $1 / 15 / 4$ & & $1 / 0 / 10 / 1 / 1$ & & \multicolumn{2}{|c|}{$0 / 1 / 10 / 2 / 0$} & $0 / 12 / 13$ & $10 / 0 / 6$ & $90 / 88 / 81$ \\
\hline Chloramphenicol $^{4}$ & $8 / 8 / 8$ & $8 / 16 / 64$ & & & & & & $1 / 0 / 0$ & & $2 / 1 / 00 / 8 / 5$ & $7 / 21 / 6$ & \multicolumn{2}{|c|}{$0 / 1 / 40 / 2 / 00 / 1 / 3$} & $0 / 9 / 19$ & $0 / 3 / 13$ & $100 / 88 / 69$ \\
\hline Tetracycline ${ }^{2}$ & $0.5 / 0.5 / 0.5$ & $64 / 64 / 64$ & & & $0 / 2 / 0$ & & $3 / 11 / 3$ & $211 / 6$ & $1 / 3 / 2$ & & $0 / 1 / 0$ & \multicolumn{3}{|c|}{$1 / 1 / 0$ 0/0/1 3/5/3 0/0/1 50/29/44 } & $20 / 32 / 38$ & $30 / 38 / 19$ \\
\hline
\end{tabular}

R, resistant; I, Intermediate; S, susceptible; MIC, minimum inhibitory concentration.

$\mathrm{MIC}_{50}$, lowest concentration to inhibit $50 \%$ of bacteria; $\mathrm{MIC}_{90}$, lowest concentration to inhibit $90 \%$ of bacteria.

The dilution ranges tested are those contained in the white area. Values above this range indicate MIC values higher than the highest concentration within the range. Values below this range indicate MIC values lower than the lowest concentration within the range. Breakpoints are employed according to VET01S document. When available, susceptible and resistance breakpoints are indicated in vertical dotted and solid lines respectively. For antibiotics without intermediate zone, a single vertical solid line is indicated. For oxacillin, breakpoint of $S$. aureus is indicated in vertical doubled solid line and breakpoint of $S$. (pseud) intermedius and $S$. hyicus is in vertical doubled dotted line.

${ }^{1} 10$ isolates from stray cats, 31 isolates from hospital-admitted cat, and 16 isolates from hospital staff

${ }^{2}$ Only CLSI breakpoint for dog isolates available.

${ }^{3}$ Indicates isolates numbers from stray cat, hospital-admitted cat and hospital staff, respectively.

${ }^{4}$ The breakpoints derived from human breakpoints used [18].

${ }^{5}$ No CLSI breakpoint available.

Table 3. MICs of 11 antimicrobial agents against 454 Coagulase-negative Staphylococci ${ }^{1}$ isolated in this study. 


\begin{tabular}{|c|c|c|c|c|c|c|c|c|c|c|c|c|c|c|c|c|c|c|c|c|}
\hline \multirow[t]{2}{*}{ Antimicrobials } & \multirow{2}{*}{$\begin{array}{l}\mathrm{MIC}_{50} \\
(\mu \mathrm{g} / \mathrm{ml})\end{array}$} & \multirow{2}{*}{$\begin{array}{l}\mathrm{MIC}_{90} \\
(\mu \mathrm{g} / \mathrm{ml})\end{array}$} & \multicolumn{15}{|c|}{ MIC values $(\mu \mathrm{g} / \mathrm{ml})$} & \multirow[b]{2}{*}{$\% \mathrm{R}$} & \multirow[b]{2}{*}{$\% 1$} & \multirow[b]{2}{*}{$\% S$} \\
\hline & & & $\overline{0.008}$ & 0.015 & 0.03 & 0.06 & 0.12 & 0.25 & 0.5 & 1 & 2 & 4 & 8 & 16 & 32 & 64 & 128 & & & \\
\hline$\overline{\text { Ampicillin }^{2}}$ & $0.06 / 0.25 / 0.5^{3}$ & $1 / 2 / 16$ & & $8 / 11 / 4$ & & $25 / 45 / 14$ & $10 / 44 / 21$ & $8 / 50 / 22$ & $5 / 31 / 19$ & $4 / 16 / 21$ & $4 / 16 / 21$ & $0 / 5 / 15$ & $0 / 3 / 6$ & $0 / 2 / 7$ & $0 / 6 / 7$ & $0 / 1 / 3$ & & $20 / 35 / 62$ & $20 / 0 / 0$ & $80 / 65 / 38$ \\
\hline Penicillin 4 & $0.06 / 0.25 / 1$ & $1 / 2 / 16$ & $10 / 19 / 2$ & & $11 / 13 / 6$ & $14 / 36 / 15$ & $7 / 23 / 9$ & $8 / 51 / 22$ & $3 / 32 / 21$ & $5 / 16 / 18$ & $3 / 18 / 24$ & $1 / 7 / 16$ & $1 / 6 / 9$ & $1 / 5 / 6$ & $0 / 1 / 6$ & $0 / 3 / 6$ & & $34 / 60 / 80$ & $0 / 0 / 0$ & $66 / 40 / 20$ \\
\hline Oxacillin ${ }^{4}$ & $0.25 / 0.25 / 2$ & $2 / 8 / \geq 64$ & & $2 / 5 / 0$ & & $14 / 28 / 8$ & $15 / 47 / 24$ & $11 / 44 / 14$ & $7 / 34 / 10$ & $8 / 21 / 13$ & $3 / 14 / 14$ & $0 / 7 / 27$ & $1 / 8 / 19$ & $1 / 5 / 6$ & $0 / 8 / 4$ & $2 / 9 / 21$ & & $34 / 46 / 71$ & $10 / 0 / 0$ & $66 / 54 / 29$ \\
\hline Amox/clav & $0.12 / 0.12 / 0.5$ & $1 / 0.5 / 2$ & & & $27 / 48 / 16$ & $1 / 3 / 1$ & $19 / 73 / 21$ & $10 / 51 / 34$ & $3 / 26 / 28$ & $2 / 19 / 33$ & $0 / 2 / 11$ & $2 / 3 / 8$ & $0 / 3 / 3$ & $0 / 0 / 2$ & $0 / 2 / 3$ & & & 6/13/38 & $5 / 11 / 18$ & $89 / 76 / 45$ \\
\hline Cephalexin $^{5}$ & $2 / 2 / 8$ & $16 / 16 / 32$ & & & & $0 / 2 / 2$ & & $0 / 1 / 3$ & $2 / 3 / 1$ & $17 / 41 / 10$ & $31 / 102 / 38$ & $87 / 39 / 19$ & $3 / 13 / 19$ & $2 / 11 / 31$ & $1 / 6 / 20$ & $1 / 3 / 3$ & $0 / 9 / 9$ & & & \\
\hline Gentamicin ${ }^{4}$ & $\leq 0.06 / \leq 0.06 / 1$ & $16 / 16 / \geq 64$ & & & & $54 / 158 / 61$ & $0 / 2 / 0$ & $3 / 14 / 12$ & $0 / 5 / 4$ & $1 / 2 / 4$ & $1 / 3 / 1$ & $0 / 6 / 9$ & $0 / 14 / 9$ & $3 / 5 / 10$ & $1 / 13 / 20$ & $1 / 8 / 30$ & & $8 / 11 / 38$ & $0 / 6 / 6$ & $92 / 83 / 57$ \\
\hline Clindamycin ${ }^{2}$ & $0.12 / 0.12 / 0.12$ & $1 / 0.5 / 2$ & & $1 / 12 / 7$ & & $26 / 67 / 49$ & $19 / 90 / 70$ & $5 / 21 / 4$ & $9 / 14 / 6$ & $2 / 8 / 5$ & $0 / 2 / 4$ & $0 / 6 / 4$ & $1 / 2 / 2$ & $1 / 0 / 2$ & & $0 / 8 / 7$ & & $3 / 7 / 9$ & $3 / 4 / 6$ & $94 / 89 / 85$ \\
\hline Enrofloxacin & $0.12 / 0.12 / 0.25$ & $0.25 / 1 / 4$ & & $2 / 681$ & & $25 / 58 / 24$ & $21 / 89 / 50$ & $14 / 39 / 23$ & $2 / 14 / 14$ & $0 / 12 / 26$ & $0 / 4 / 4$ & $0 / 2 / 3$ & & $0 / 0 / 7$ & $0 / 4 / 8$ & $0 / 2 / 0$ & & $0 / 4 / 11$ & $0 / 7 / 19$ & $100 / 90 / 70$ \\
\hline Marbofloxacin & $0.25 / 0.05 / 0.25$ & $0.5 / 1 / 8$ & & & $0 / 3 / 5$ & $0 / 1 / 0$ & $16 / 30 / 7$ & $42 / 134 / 76$ & $6 / 35 / 25$ & $0 / 16 / 28$ & $0 / 3 / 4$ & & $0 / 1 / 7$ & $0 / 2 / 6$ & $0 / 3 / 5$ & $0 / 2 / 0$ & & $0 / 4 / 11$ & $0 / 1 / 3$ & $100 / 95 / 86$ \\
\hline Chloramphenicol ${ }^{4}$ & $4 / 4 / 4$ & $8 / 8 / 8$ & & & & & & & $0 / 2 / 1$ & & $10 / 37 /$ & $42 / 125 / 94$ & $11 / 54 / 42$ & $0 / 1 / 2$ & $1 / 3 / 1$ & $0 / 5 / 4$ & $0 / 3 / 0$ & $2 / 5 / 3$ & $0 / 0 / 1$ & 98/95/96 \\
\hline Tetracycline ${ }^{2}$ & $0.25 / 0.25 / 2$ & $16 / 32 / \geq 128$ & & & & $9 / 21 / 1$ & & $32 / 96 / 40$ & $17 / 58 / 24$ & $41 / 15 / 7$ & $1 / 5 / 12$ & $0 / 4 / 3$ & $0 / 0 / 4$ & $1 / 2 / 5$ & $2 / 7 / 9$ & $1 / 15 / 25$ & $50 / 7 / 30$ & 0 9/24/59 & $27 / 25 / 15$ & $64 / 51 / 26$ \\
\hline
\end{tabular}

R, resistant; I, Intermediate; S, susceptible; MIC, minimum inhibitory concentration.

$\mathrm{MIC}_{50}$, lowest concentration to inhibit $50 \%$ of bacteria; $\mathrm{MIC}_{90}$, lowest concentration to inhibit $90 \%$ of bacteria.

The dilution ranges tested are those contained in the white area. Values above this range indicate MIC values higher than the highest concentration within the range. Values below this range indicate MIC values lower than the lowest concentration within the range. Breakpoints are employed according to VET01S document. When available, susceptible and resistance breakpoints are indicated in vertical dotted and solid lines respectively. For antibiotics without intermediate zone, a single vertical solid line is indicated.

${ }^{1} 10$ isolates from stray cats, 31 isolates from hospital-admitted cat, and 16 isolates from hospital staff

${ }^{2}$ Only CLSI breakpoint for dog isolates available.

${ }^{3}$ Indicates isolates numbers from stray cat, hospital-admitted cat and hospital staff, respectively.

${ }^{4}$ The breakpoints derived from human breakpoints used [18].

${ }^{5}$ No CLSI breakpoint available.

Table 4. MICs of 13 antimicrobial agents against 278 Enterobacteriaceae $^{1}$ isolated in this study 


\begin{tabular}{|c|c|c|c|c|c|c|c|c|c|c|c|c|c|c|c|c|c|c|c|c|c|c|}
\hline \multicolumn{2}{|c|}{ Antimicrobials $\mathrm{MIC}_{50}$} & \multirow{2}{*}{$\begin{array}{l}\mathrm{MIC}_{90} \\
(\mathrm{\mu g} / \mathrm{ml})\end{array}$} & \multicolumn{17}{|c|}{ MIC values $(\mu \mathrm{g} / \mathrm{ml})$} & \multirow[b]{2}{*}{$\% \mathrm{R}$} & \multirow[b]{2}{*}{$\% 1$} & \multirow[b]{2}{*}{$\% S$} \\
\hline & $(\mu \mathrm{g} / \mathrm{ml})$ & & 0.004 & 0.008 & 0.015 & 0.03 & 0.06 & 0.12 & 0.25 & 0.5 & 1 & 2 & 4 & 8 & 16 & 32 & 64 & 128 & 256 & & & \\
\hline Ampicillin $^{2}$ & $4 / 4 / 4^{3}$ & $\begin{array}{c}\geq 128 / \geq 128 / \\
\geq 128\end{array}$ & & & & & $0 / 2 / 0$ & & $0 / 0 / 1$ & & $0 / 1 / 1$ & $6 / 37 / 9$ & $23 / 62 / 14$ & $2 / 16 / 2$ & $1 / 6 / 6$ & $1 / 9 / 1$ & $2 / 5 / 5$ & $4 / 51 / 11$ & & $100 / 99 / 98$ & $30 / 0 / 0$ & $0 / 1 / 2$ \\
\hline Amox/clav & $8 / 8 / 16$ & $32 / 32 / 64$ & & & & $0 / 1 / 1$ & & $0 / 2 / 0$ & $0 / 0 / 1$ & $1 / 1 / 0$ & $0 / 1 / 1$ & $0 / 13 / 1$ & $15 / 50 / 12$ & $13 / 65 / 4$ & $4 / 25 / 7$ & 3/13/3 & 3 0/8/17 & $3 / 10 / 3$ & & $97 / 98 / 96$ & $3 / 1 / 0$ & $0 / 2 / 4$ \\
\hline Cephalexin ${ }^{4}$ & $8 / 8 / 32$ & $\begin{array}{c}\geq 128 / \geq 128 / \\
\geq 128\end{array}$ & & & & & & & & & $0 / 2 / 1$ & $1 / 1 / 0$ & $5 / 31 / 1$ & $21 / 81 / 12$ & $27 / 35 / 7$ & $1 / 9 / 11$ & 1 1/1/11 & $3 / 29 / 7$ & & & & \\
\hline Cephalothin $^{2}$ & $8 / 8 / 32$ & $\geq 64 / \geq 64 / \geq 64$ & & & & & & $0 / 1 / 0$ & & $0 / 1 / 1$ & $0 / 1 / 1$ & $1 / 5 / 1$ & $10 / 38 / 3$ & $20 / 81 / 9$ & $3 / 20 / 3$ & $30 / 8 / 3$ & $5 / 34 / 29$ & & & $72 / 76 / 88$ & $26 / 20 / 6$ & $3 / 4 / 6$ \\
\hline Cefoxitin $^{5}$ & $4 / 4 / 16$ & $\begin{array}{c}16 / \geq 128 / \\
\geq 128\end{array}$ & & & & & & & & & $1 / 1 / 0$ & $5 / 16 / 2$ & $14 / 88 / 8$ & $13 / 46 / 9$ & $1 / 7 / 13$ & $30 / 3 / 2$ & $0 / 3 / 1$ & $5 / 25 / 15$ & & $13 / 16 / 36$ & $3 / 4 / 26$ & $85 / 8038 /$ \\
\hline Ceftiofur $^{6}$ & $0.5 / 0.5 / 0.5$ & $2 / 4 / 2$ & & & & $0 / 3 / 1$ & & $2 / 3 / 0$ & $6 / 40 / 17$ & $22 / 76 / 14$ & 4 6/38/12 & $2 / 8 / 3$ & $0 / 2 / 0$ & $0 / 3 / 1$ & $0 / 1 / 0$ & $1 / 0 / 2$ & $0 / 1 / 0$ & $0 / 14 / 0$ & & $3 / 10 / 6$ & $0 / 1 / 0$ & $97 / 89 / 94$ \\
\hline Ceftriaxone $^{5}$ & $\leq 0.015$ & $1 / 4 / 0.12$ & & & $22 / 124 / 36$ & & $13 / 28 / 8$ & 8 1/10/2 & $1 / 1 / 0$ & $0 / 3 / 0$ & $1 / 2 / 0$ & & $0 / 3 / 0$ & $0 / 1 / 1$ & $0 / 1 / 2$ & $1 / 0 / 0$ & $0 / 16 / 1$ & & & $3 / 11 / 8$ & $0 / 0 / 0$ & $97 / 89 / 92$ \\
\hline Gentamicin $^{2}$ & $1 / 1 / 0.5$ & $2 / 2 / 1$ & & & & $0 / 3 / 1$ & & & $1 / 0 / 1$ & $4 / 18 / 25$ & $30 / 124 / 18$ & $83 / 29 / 1$ & $0 / 6 / 1$ & & $0 / 16 / 0$ & & $1 / 1 / 3$ & $0 / 5 / 0$ & & $3 / 5 / 6$ & $0 / 3 / 2$ & $97 / 92 / 92$ \\
\hline Imipenem 5 & $0.25 / 0.25 / 0.5$ & $0.5 / 0.5 / 1$ & & & $0 / 6 / 3$ & & $2 / 3 / 0$ & $15 / 58 / 3$ & 3 14/84/13 & $37 / 23 / 18$ & $0 / 9 / 11$ & $0 / 6 / 1$ & $1 / 0 / 1$ & & & & & & & $3 / 0 / 2$ & $0 / 3 / 2$ & $97 / 97 / 96$ \\
\hline Meropenem ${ }^{5}$ & $\begin{array}{c}\leq 0.015 / \\
\leq 0.015 / 0.06\end{array}$ & $0.12 / 0.25 / 0.25$ & & & $27 / 132 / 16$ & & $4 / 15 / 18$ & $85 / 16 / 3$ & $3 / 21 / 8$ & $0 / 1 / 3$ & $0 / 1 / 0$ & $0 / 0 / 1$ & & $0 / 3 / 1$ & & & & & & $0 / 2 / 2$ & $0 / 0 / 2$ & $100 / 98 / 96$ \\
\hline Enrofloxacin & $0.015 / 0.03 / 0.06$ & $0.25 / 0.5 / 0.5$ & & & $19 / 77 / 9$ & $14 / 57 / 16$ & $2 / 14 / 14$ & 4 0/15/5 & $1 / 7 / 0$ & $1 / 3 / 2$ & $1 / 5 / 2$ & $0 / 0 / 1$ & $1 / 0 / 0$ & & $0 / 8 / 1$ & $0 / 3 / 0$ & & & & $3 / 6 / 2$ & $33 / 3 / 6$ & $95 / 92 / 92$ \\
\hline Marbofloxacin & $0.015 / 0.015 / 0.03$ & $0.12 / 0.5 / 0.5$ & $0 / 5 / 0$ & & $24 / 104 / 16$ & $10 / 37 / 10$ & $2 / 7 / 16$ & $0 / 4 / 1$ & $1 / 12 / 1$ & $1 / 6 / 3$ & $0 / 2 / 2$ & $1 / 1 / 1$ & $0 / 1 / 0$ & $0 / 6 / 0$ & $0 / 1 / 0$ & $0 / 3 / 0$ & & & & $0 / 6 / 0$ & $3 / 1 / 2$ & $97 / 94 / 98$ \\
\hline Tetracycline $^{5}$ & $2 / 2 / 2$ & $16 / 128 / 128$ & & & & & $0 / 1 / 1$ & & $0 / 2 / 0$ & $1 / 3 / 1$ & $14 / 53 / 5$ & $21 / 85 / 18$ & $31 / 3 / 1$ & $0 / 1 / 2$ & & $0 / 1 / 1$ & $1 / 10 / 13$ & 3 0/14/7 & $1 / 16 / 1$ & $15 / 22 / 44$ & $0 / 1 / 4$ & $95 / 78 / 52$ \\
\hline
\end{tabular}

R, resistant; I, Intermediate; S, susceptible; MIC, minimum inhibitory concentration.

$\mathrm{MIC}_{50}$, lowest concentration to inhibit $50 \%$ of bacteria; $\mathrm{MIC}_{90}$, lowest concentration to inhibit $90 \%$ of bacteria.

The dilution ranges tested are those contained in the white area. Values above this range indicate MIC values higher than the highest concentration within the range. Values below this range indicate MIC values lower than the lowest concentration within the range. Breakpoints are employed according to VET01S document. When available, susceptible and resistance breakpoints are indicated in vertical dotted and solid lines respectively.

${ }^{1} 39$ isolates from stray cats, 189 isolates from hospital-admitted cat, and 50 isolates from hospital staff

${ }^{2}$ Only CLSI breakpoint for dog isolates available.

${ }^{3}$ Indicates isolates numbers from stray cat, hospital-admitted cat, and hospital staff, respectively.

${ }^{4}$ No CLSI breakpoint available.

${ }^{5}$ The breakpoints derived from human breakpoints used [18].

${ }^{6}$ Only CLSI breakpoint for cattle isolates available.

Table 5. MICs of 10 antimicrobial agents against 226 Enterococcus spp. ${ }^{1}$ isolated in this study 


\begin{tabular}{|c|c|c|c|c|c|c|c|c|c|c|c|c|c|c|c|c|c|c|}
\hline \multirow[t]{2}{*}{ Antimicrobials } & \multirow{2}{*}{$\begin{array}{l}\mathrm{MIC}_{50} \\
(\mu \mathrm{g} / \mathrm{ml})\end{array}$} & \multirow{2}{*}{$\begin{array}{l}\mathrm{MIC}_{90} \\
(\mu \mathrm{g} / \mathrm{ml})\end{array}$} & \multicolumn{13}{|c|}{ MIC values $(\mu \mathrm{g} / \mathrm{ml})$} & \multirow[b]{2}{*}{$\% \mathrm{R}$} & \multirow[b]{2}{*}{$\% 1$} & \multirow[b]{2}{*}{$\% \mathrm{~S}$} \\
\hline & & & 0.06 & 0.12 & 0.25 & 0.5 & 1 & 2 & 4 & 8 & 16 & 32 & 64 & 128 & 256 & & & \\
\hline Ampicillin & $2 / 2 / 1^{2}$ & $8 / 64 / 4$ & & $0 / 4 / 1$ & & $8 / 10 / 4$ & $11 / 44 / 5$ & $50 / 62 / 7$ & $2 / 9 / 3$ & $1 / 4 / 0$ & $0 / 1 / 0$ & & $0 / 2 / 0$ & $0 / 4 / 0$ & $5 / 9 / 0$ & $9 / 11 / 0$ & $0 / 0 / 0$ & $91 / 89 / 100$ \\
\hline Penicillin & $4 / 4 / 4$ & $64 / 32 / 8$ & & & $3 / 7 / 1$ & & $5 / 6 / 3$ & $5 / 13 / 2$ & $23 / 58 / 9$ & $12 / 38 / 4$ & 4 3/8/1 & $0 / 6 / 0$ & $1 / 2 / 0$ & $5 / 11 / 0$ & & $16 / 18 / 5$ & $0 / 0 / 0$ & $84 / 82 / 95$ \\
\hline Vancomycin & $1 / 1 / 1$ & $4 / 4 / 2$ & & $0 / 7 / 1$ & & $14 / 22 / 5$ & $530 / 63 / 7$ & $77 / 28 / 5$ & $1 / 21 / 1$ & $5 / 8 / 1$ & & & & & & $0 / 0 / 0$ & $9 / 5 / 5$ & $91 / 95 / 95$ \\
\hline Erythromycin & $8 / 2 / 2$ & $\geq 256 / \geq 256 / 4$ & & $16 / 34 / 7$ & & $1 / 9 / 1$ & $5 / 17 / 0$ & $4 / 18 / 4$ & $2 / 13 / 3$ & $1 / 1 / 2$ & & $4 / 1 / 0$ & $0 / 1 / 0$ & $0 / 3 / 0$ & $24 / 52 / 3$ & $351 / 39 / 25$ & 5 19/32/35 & $530 / 29 / 40$ \\
\hline Chloramphenicol & | 8/8/4 & $64 / 32 / 16$ & & & & $0 / 2 / 1$ & & $5 / 15 / 0$ & $20 / 49 / 9$ & $8 / 38 / 7$ & $12 / 19 / 2$ & $25 / 15 / 0$ & $7 / 11 / 1$ & & & $21 / 17 / 5$ & $21 / 13 / 10$ & 0 58/70/85 \\
\hline Ciprofloxacin & $1 / 2 / 1$ & $4 / 4 / 2$ & & & $14 / 35 / 7$ & & $22 / 39 / 7$ & $712 / 45 / 4$ & $4 / 4 / 2$ & $1 / 5 / 0$ & & $1 / 6 / 0$ & $3 / 0 / 0$ & & & $16 / 20 / 10$ & $021 / 30 / 20$ & 0 63/50/70 \\
\hline Tetracycline & $32 / 32 / 1$ & $\geq 256 / 128 / 128$ & & $8 / 29 / 3$ & & $4 / 18 / 6$ & $1 / 4 / 1$ & $1 / 2 / 0$ & $2 / 1 / 0$ & $2 / 2 / 0$ & $1 / 4 / 1$ & $13 / 25 / 4$ & $46 / 18 / 1$ & $11 / 36 / 4$ & $4 / 10 / 0$ & $68 / 62 / 50$ & $04 / 1 / 0$ & $28 / 36 / 50$ \\
\hline Minocycline & $16 / 16 / 0.25$ & $32 / 32 / 16$ & $14 / 42 / 9$ & & $2 / 5 / 2$ & $1 / 10 / 1$ & $0 / 1 / 1$ & & $3 / 5 / 0$ & $3 / 4 / 0$ & $15 / 42 / 5$ & 5 19/39/2 & $20 / 1 / 0$ & & & $60 / 55 / 35$ & $55 / 3 / 0$ & $35 / 42 / 65$ \\
\hline Streptomycin ${ }^{3}$ & & & & & & $2(<2,000)$ & & & & & & $5(>2,00$ & & & & $9 / 9 / 5$ & & \\
\hline Gentamicin $^{4}$ & & & & & & $7(<500)$ & & & & & & $10(>50$ & & & & $18 / 17 / 5$ & & \\
\hline
\end{tabular}

R, resistant; I, Intermediate; S, susceptible; MIC, minimum inhibitory concentration.

$\mathrm{MIC}_{50}$, lowest concentration to inhibit $50 \%$ of bacteria; $\mathrm{MIC}_{90}$, lowest concentration to inhibit $90 \%$ of bacteria.

The dilution ranges tested are those contained in the white area. Values above this range indicate MIC values higher than the highest concentration within the range. Values below this range indicate MIC values lower than the lowest concentration within the range. The breakpoints derived from human breakpoints used. When available, susceptible and resistance breakpoints are indicated in vertical dotted and solid lines respectively. For antibiotics without intermediate zone, a single vertical solid line is indicated.

${ }^{1} 57$ isolates from stray cats, 149 isolates from hospital-admitted cat, and 20 isolates from hospital staff

${ }^{2}$ Indicates isolates numbers from stray cat, hospital-admitted cat, and hospital staff, respectively.

${ }^{3}$ High-level resistance to streptomycin $(2,000 \mu \mathrm{g} / \mathrm{ml})$.

${ }^{4}$ High-level resistance to gentamicin $(500 \mu \mathrm{g} / \mathrm{ml})$.

Table 6. MICs of 11 antimicrobial agents against 110 mecA-positive staphylococci isolated in this study.

\begin{tabular}{|c|c|c|c|c|c|c|c|c|c|c|c|c|c|c|}
\hline \multirow[t]{3}{*}{ Antimicrobials } & \multicolumn{5}{|c|}{ stray cats (7) } & \multicolumn{5}{|c|}{ hospital-admitted cats (32) } & \multicolumn{4}{|c|}{ veterinary staff (71) } \\
\hline & $\mathrm{MIC}_{50}$ & $\mathrm{MIC}_{90}$ & $\% \mathrm{R}$ & $\% 1$ & $\% \mathrm{~S}$ & $\mathrm{MIC}_{50}$ & $\mathrm{MIC}_{90}$ & $\% R$ & $\% 1$ & $\% \mathrm{~S}$ & $\mathrm{MIC}_{50}$ & $\mathrm{MIC}_{90}$ & $\% \mathrm{R} \% \mathrm{I}$ & $\% \mathrm{~S}$ \\
\hline & \multicolumn{4}{|c|}{$(\mu \mathrm{g} / \mathrm{ml})(\mu \mathrm{g} / \mathrm{ml})$} & & $(\mu \mathrm{g} / \mathrm{ml})$ & $(\mu \mathrm{g} / \mathrm{ml})$ & & & & \multicolumn{4}{|c|}{$(\mu \mathrm{g} / \mathrm{ml})(\mu \mathrm{g} / \mathrm{ml})$} \\
\hline$\overline{\text { Ampicillin }^{1}}$ & 2 & 32 & 85.7 & & 14.3 & & 32 & 84.4 & 0 & 15.6 & 2 & 16 & 88.70 & 11.3 \\
\hline Penicillin ${ }^{2}$ & 1 & $\geq 64$ & 85.7 & & 14.3 & & 16 & 96.9 & 0 & 3.1 & 2 & 32 & 98.60 & 1.4 \\
\hline Oxacillin ${ }^{2}$ & 2 & $\geq 64$ & 100 & 0 & 0 & 16 & $\geq 64$ & 100 & 0 & 0 & 4 & $\geq 64$ & 1000 & 0 \\
\hline Amox/clav & 1 & 8 & 57. & 128.6 & 514.3 & & 8 & 53.1 & 37.5 & 9.4 & 1 & 4 & 66.219 .7 & 714.1 \\
\hline Cephalexin $^{3}$ & 16 & 64 & & & & 16 & 64 & & & & 16 & 64 & & \\
\hline Gentamicin $^{2}$ & 0.25 & 32 & 28 & & 71.4 & & $\geq 64$ & 40.6 & 18.8 & 40.6 & 32 & $\geq 64$ & 60.65 .6 & 33.8 \\
\hline Clindamycin $^{1}$ & 0.5 & 16 & 14.3 & B 28.6 & 57.1 & 10.12 & 4 & 12.5 & 0 & 87.5 & 0.12 & 8 & 15.55 .6 & 78.9 \\
\hline Enrofloxacin & 0.25 & 2 & 0 & 14.3 & 85.7 & 70.25 & 4 & 12.5 & 15.6 & 71.9 & 0.5 & 16 & 14.132 .4 & 453.5 \\
\hline Marbofloxacin & 0.25 & 2 & 0 & 14.3 & 85.7 & 70.5 & 16 & 12.5 & 0 & 87.5 & 0.5 & 8 & 14.15 .6 & 80.3 \\
\hline Chloramphenicol $^{2}$ & & 32 & 14.3 & & 85.7 & & 8 & 3.1 & 0 & 96.9 & 4 & 8 & $2.8 \quad 1.4$ & 95.8 \\
\hline Tetracycline ${ }^{a}$ & 32 & 64 & 57. & 128.6 & 514.3 & & 64 & 53.1 & 25 & 21.9 & 64 & $\geq 128$ & 88.75 .6 & 5.6 \\
\hline
\end{tabular}


R, resistant; I, Intermediate; S, susceptible; MIC, minimum inhibitory concentration.

$\mathrm{MIC}_{50}$, lowest concentration to inhibit $50 \%$ of bacteria; $\mathrm{MIC}_{90}$, lowest concentration to inhibit $90 \%$ of bacteria.

Breakpoints are employed according to VET01S document.

${ }^{1}$ Only CLSI breakpoint for dog isolates available.

${ }^{2}$ The breakpoints derived from human breakpoints used [18].

${ }^{3}$ No CLSI breakpoint available.

Table 7. The most prevalent resistance profile per antimicrobial category found in Coagulase-negative Staphylococci isolated in this study based on CLSI human clinical breakpoint data.

\begin{tabular}{|c|c|c|c|c|}
\hline \multirow{2}{*}{$\begin{array}{l}\text { No. antimicrobial } \\
\text { category }\end{array}$} & \multirow{2}{*}{$\begin{array}{l}\text { No. of isolates } \\
(\%)\end{array}$} & \multicolumn{3}{|c|}{ Resistance pattern (no. of isolates) } \\
\hline & & stray cats $(n=64)$ & hospital-admitted cats $(\mathrm{n}=230)$ & veterinary staff $(n=160)$ \\
\hline All susceptible & $115(25.3)$ & 31 & 64 & 20 \\
\hline 1 & $109(24.0)$ & OXA (11) & BLA (37) & BLA (18) \\
\hline 2 & $80(17.6)$ & BLA-OXA (4) & BLA-OXA (38) & BLA-OXA (15) \\
\hline 3 & $54(11.9)$ & AMG-BLA-OXA (1) & BLA-BLI-OXA (14) & BLA-BLI-OXA (9) \\
\hline 4 & $38(8.4)$ & BLA-BLI-OXA-TET (2) & AMG-BLA-OXA-TET (3) & AMG-BLA-OXA-TET (8) \\
\hline 5 & $39(8.6)$ & - & AMG-BLA-BLI-OXA-TET (4) & AMG-BLA-BLI-OXA-TET (24) \\
\hline 6 & $18(4.0)$ & $\begin{array}{l}\text { AMG-BLA-BLI-OXA-LIN-TET } \\
\text { (1) }\end{array}$ & AMG-BLA-BLI-OXA-FQN-TET (2) & AMG-BLA-BLI-OXA-FQN-TET (6) \\
\hline 7 & $3(0.7)$ & - & $\begin{array}{l}\text { AMG-BLA-BLI-OXA-LIN-PNC-TET } \\
\text { (2) }\end{array}$ & $\begin{array}{l}\text { AMG-BLA-BLI-OXA-FQN-LIN-TET } \\
\text { (2) }\end{array}$ \\
\hline Non-MDR & $304(67.0)$ & $57\left(89.1^{1}\right)$ & $179\left(77.8^{1}\right)$ & $68\left(42.5^{1}\right)$ \\
\hline MDR & $150(33.0)$ & $7\left(10.9^{1}\right)$ & $51\left(22.2^{1}\right)$ & $92\left(57.5^{1}\right)$ \\
\hline
\end{tabular}

Antimicrobial categories included: aminoglycosides, AMG (gentamicin); $\beta$-lactam groups, BLA (ampicillin and penicillin); Oxacillin, OXA; $\beta$-lactam/ $\beta$ lactamase inhibitor combination, BLI (amoxicillin/clavulanate); fluoroquinolones, FQN (enrofloxacin and marbofloxacin); lincosamide, LIN (clindamycin); phenicol, PNC (chloramphenicol); and tetracycline, TET

${ }^{1}$ Percentages

Table 8. The most prevalent resistance profile per antimicrobial category found in Enterobacteriaceae isolated in this study based on CLSI human clinical breakpoint data.

\begin{tabular}{|c|c|c|c|c|}
\hline \multirow[t]{2}{*}{ No. antimicrobial category } & \multirow[t]{2}{*}{ No. of isolates (\%) } & \multicolumn{3}{|c|}{ Resistance pattern (no. of isolates) } \\
\hline & & stray cats $(n=39)$ & hospital-admitted cats $(\mathrm{n}=189)$ & veterinary staff $(n=50)$ \\
\hline All susceptible & $135(48.6)$ & 29 & 99 & 7 \\
\hline 1 & $32(11.5)$ & BLA (2) & $\operatorname{BLA}(9)$ & BLA (3) \\
\hline 2 & $40(14.4)$ & BLA-BLI (1) & BLA-TET (8) & GC-TET (9) \\
\hline 3 & $48(17.3)$ & BLA-BLI-GC (1) & BLA-BLI-GC (11) & BLA-BLI-GC (9) \\
\hline 4 & $12(4.3)$ & BLA-BLI-GC-CPM (1) & BLA-BLI-GC-3GC (3) & AMG-BLA-BLI-TET (1) \\
\hline 5 & $9(3.2)$ & BLA-BLI-GC-3GC-TET (1) & BLA-BLI-GC-3GC-TET (4) & - \\
\hline 6 & $2(0.7)$ & - & AMG-BLA- GC-3GC-FQN-TET (1) & - \\
\hline Non-MDR & $207(74.5)$ & $35\left(89.7^{1}\right)$ & $144\left(76.2^{1}\right)$ & $28\left(56.0^{1}\right)$ \\
\hline MDR & $71(25.5)$ & $4\left(10.3^{1}\right)$ & $45\left(23.8^{1}\right)$ & $22\left(44.0^{1}\right)$ \\
\hline
\end{tabular}

Antimicrobial categories included: aminoglycosides, AMG (gentamicin); $\beta$-lactam group, BLA (ampicillin); $\beta$-lactam/ $\beta$-lactamase inhibitor combination, BLI (amoxicillin/clavulanate); $1^{\text {st }}$ and $2^{\text {nd }}$ generation cephalosporins, GC (cephalexin, cephalothin and cefoxitin); $3^{\text {rd }}$ generation cephalosporins, $3 \mathrm{GC}$ (ceftiofur and ceftriaxone); fluoroquinolones, FQN (enrofloxacin and marbofloxacin); carbapenems, CPM (imipenem and meropenem); and tetracycline, TET

${ }^{1}$ Percentages 
Table 9. The most prevalent resistance profile per antimicrobial category found in Enterococcus spp. isolated in this study based on CLSI human clinical breakpoint data.

\begin{tabular}{|c|c|c|c|c|}
\hline \multirow{2}{*}{$\begin{array}{l}\text { No. antimicrobial } \\
\text { category }\end{array}$} & \multirow{2}{*}{$\begin{array}{l}\text { No. of isolates } \\
(\%)\end{array}$} & \multicolumn{3}{|c|}{ Resistance pattern (no. of isolates) } \\
\hline & & stray cats $(n=57)$ & hospital-admitted cats $(n=149)$ & veterinary staff $(n=20)$ \\
\hline All susceptible & $63(27.9)$ & 13 & 43 & 7 \\
\hline 1 & $50(22.1)$ & TET (8) & TET (23) & TET (6) \\
\hline 2 & $47(20.8)$ & MAC-TET (10) & MAC-TET (16) & MAC-TET (3) \\
\hline 3 & $31(13.7)$ & MAC-PNC-TET (5) & MAC-PNC-TET (8) & - \\
\hline 4 & $14(6.2)$ & STM-MAC-PNC-TET (2) & AMG-MAC-PNC-TET (4) & AMG-MAC-PNC-TET (1) \\
\hline 5 & $12(5.3)$ & AMG-BLA-FQN-MAC-TET (2) & AMG-BLA-FQN-MAC-TET (7) & - \\
\hline 6 & $6(2.6)$ & $\begin{array}{l}\text { AMG-STM-BLA-FQN-MAC-TET } \\
\text { (2) }\end{array}$ & AMG-STM-BLA-FQN-MAC-TET (2) & - \\
\hline 7 & 3 & - & $\begin{array}{l}\text { AMG-STM-BLA-FQN-MAC-PNC-TET } \\
\text { (3) }\end{array}$ & - \\
\hline Non-MDR & $160(70.8)$ & $39\left(68.4^{1}\right)$ & $102\left(68.5^{1}\right)$ & $19\left(95.0^{1}\right)$ \\
\hline MDR & $66(29.2)$ & $18\left(31.6^{1}\right)$ & $47\left(31.5^{1}\right)$ & $1\left(5.0^{1}\right)$ \\
\hline
\end{tabular}

Antimicrobial categories included: aminoglycosides except streptomycin, AMG (gentamicin); streptomycin, STM; $\beta$-lactam groups, BLA (ampicillin and penicillin); glycopeptide, GLP (vancomycin); fluoroquinolone, FQN (ciprofloxacin); macrolide, MAC (erythromycin); phenicol, PNC (chloramphenicol); and tetracycline, TET

${ }^{1}$ Percentages

\section{Figures}

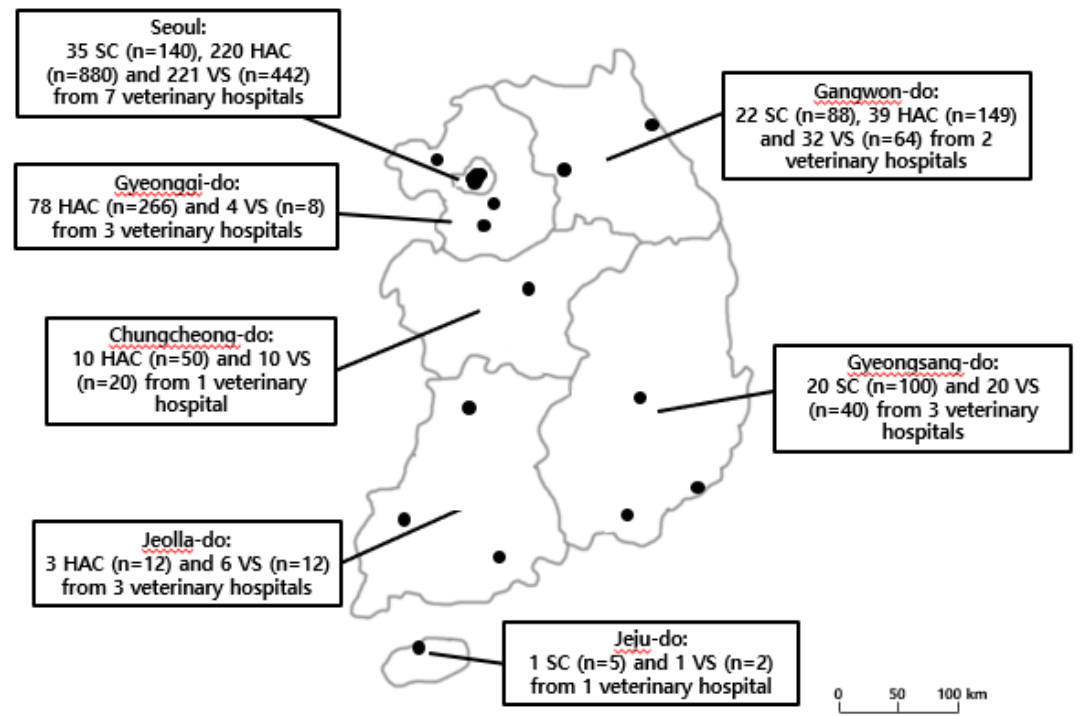

Figure 1

Geographical distribution of veterinary hospitals included in this study. The map shows physical locations of veterinary hospitals in which samples collected (closed circles) and the number of samples collected in each province. The number in parentheses indicates the total number of swab samples collected from the indicated number of individual cats or staffs. SC, stray cats; HAC, hospital-admitted cats; VS, veterinary staffs.

\section{Supplementary Files}

This is a list of supplementary files associated with this preprint. Click to download.

- FigureSFigure.pptx 